\title{
Opinion Dynamics and Sociophysics
}

\author{
D. Stauffer
}

Institute for Theoretical Physics, Cologne University, D-50923 Köln, Euroland

\author{
Article Outline \\ Glossary \\ I. Definition and Introduction \\ II. Schelling Model \\ III. Opinion Dynamics \\ IV. Languages, Hierarchies and Football \\ V. Future Directions \\ Glossary

\section{Cluster} \\ Clusters are sets of neighbouring sites of the same type.
}

\section{Ising model}

Each site carries a magnetic dipole which points up or down; neighbouring dipoles "want" to be parallel.

\section{Opinion dynamics}

How do people change opinions? Simulations usually ignore all details of the brain and represent the opinion by one or several numbers which can be changed due to contact with others.

\section{Schelling model}

People belonging do different groups may produce segretated neighbourhood just by their personal preferences, not by outside force.

\section{Sociophysics}

Application of methods from (mostly statistical) physics to human relations; can be traced centuries backwards. 
Figure 1: Ising model after 20 Glauber kinetic steps per site on a $500 \times 500$ square lattice at $k_{B} T / J=2$. We start from a random distribution of equally many black and white sites. Figs.1, 2, 3 omitted for arXiv; their small are not readable on some computers and show the standard phase separation or clustering of Ising model..

Figure 2: As Fig.1 but after 2000 instead of 20 iterations.

\section{Definition and Introduction}

The application of concepts from the natural sciences to social sciences, partly to be reviewed here, is at least 25 centuries old. Then the Greek philosopher Empedokles stated (according to J. Mimkes) than humans are like liquids: Some mix easily like wine and water, and others like oil and water refuse to mix. We start with the Schelling model of 1971 which implemented this idea, and its criticism. Then we will review opinion dynamics in large populations, summarising only shortly other aspects like self-organisation of hierarchies or competition between human languages.

Humans do not like to be treated like a number, and indeed the human brain is much more complex than a binary variable (called "spin" by physicists) which is either +1 or -1 . We do not deal here with the psychological processes of an individual but with mass psychology, and this author learned half a century ago in school that mass psychology is different from individual psychology: The law of large numbers averages out over individual fluctuations and makes general trends more clearly visible. Thus what we call today statistical physics plays a useful rule, and social scientists [1, 2] have applied it, without knowing then that they dealt with an Ising model of ferromagnets.

The astronomer Halley, best known through his comet, tried to establish mortality tables already three centuries ago. Of course, the time of death of one given individual is usually difficult to predict but averaged over millions of people the statistical offices of many countries prepare regularly life tables which tell us how probable it is for a newborn child to live up to $x$ years, provided there are no changes of the mortalities in the coming decades. Insurance for automobiles is another example: We do not want to produce 
accidents, but we know that they happen, and take precautions for their financial consequences. Thus the whole insurance industry is based on treating humans like numbers, ignoring their individuality.

Finally, human opinions are often fluctuating and ill-defined, but nevertheless in elections people cast one choice, out of a limited number of choices. And election results belong to those social data for which we have lots of accurate numbers, based on large populations.

Thus it is not at all the merit (or ignorance) of physicists which treats humans like numbers; this method has a very long tradition and is an indispensable part of modern life.

\section{Schelling Model}

\section{$2.1 \quad$ Ising simulations}

Following (but not citing) Empedokles, the later economics Nobel laureate Schelling [1] asked whether the racial segregation in American cities can emerge from intrinsic behaviour of the individual people, instead of or in addition to extrinsic reasons like discrimination, rent differences, etc. In particular, can "black" ghettos in the predominantly "white" USA arise just because people prefer to have neighbours of their own group over neighbours from the other group? In many other countries we find many other types of residential segregation, based on religion, ethnicity, .... In physics, such a process is easily simulated through the two-dimensional Ising model, as shown in Figs. 1 and 2.

In this Ising model, each site on a square lattice carries a variable $S_{i}= \pm 1$, and each pair $\left\langle i, k>\right.$ of nearest neighbours produces an "energy" $-J S_{i} S_{k}$ with some proportionality constant $J$. The total energy $E$ (= total unhappiness) is the sum of this pair energy over all neighbour pairs of the lattice. In physics, different distributions of the "spins" $S_{i}$ are realised with a probability proportional to $\exp \left(-E / k_{B} T\right)$ where $T$ is the absolute temperature and $k_{B}$ the Boltzmann constant. There is no need to worry about values for $T, k_{B}, J$ since the only relevant quantity is the ratio $k_{B} T / J$, taken as 2 in these pictures. The "Glauber" kinetics is simulated on the computer by flipping a spin if and only if a random number between 0 and 1 is smaller than the probability $\exp \left(-\Delta E / k_{B} T\right) /\left[1+\exp \left(-\Delta E / k_{B} T\right]\right.$. The Fortran program contains less than 40 lines and takes a few seconds: 


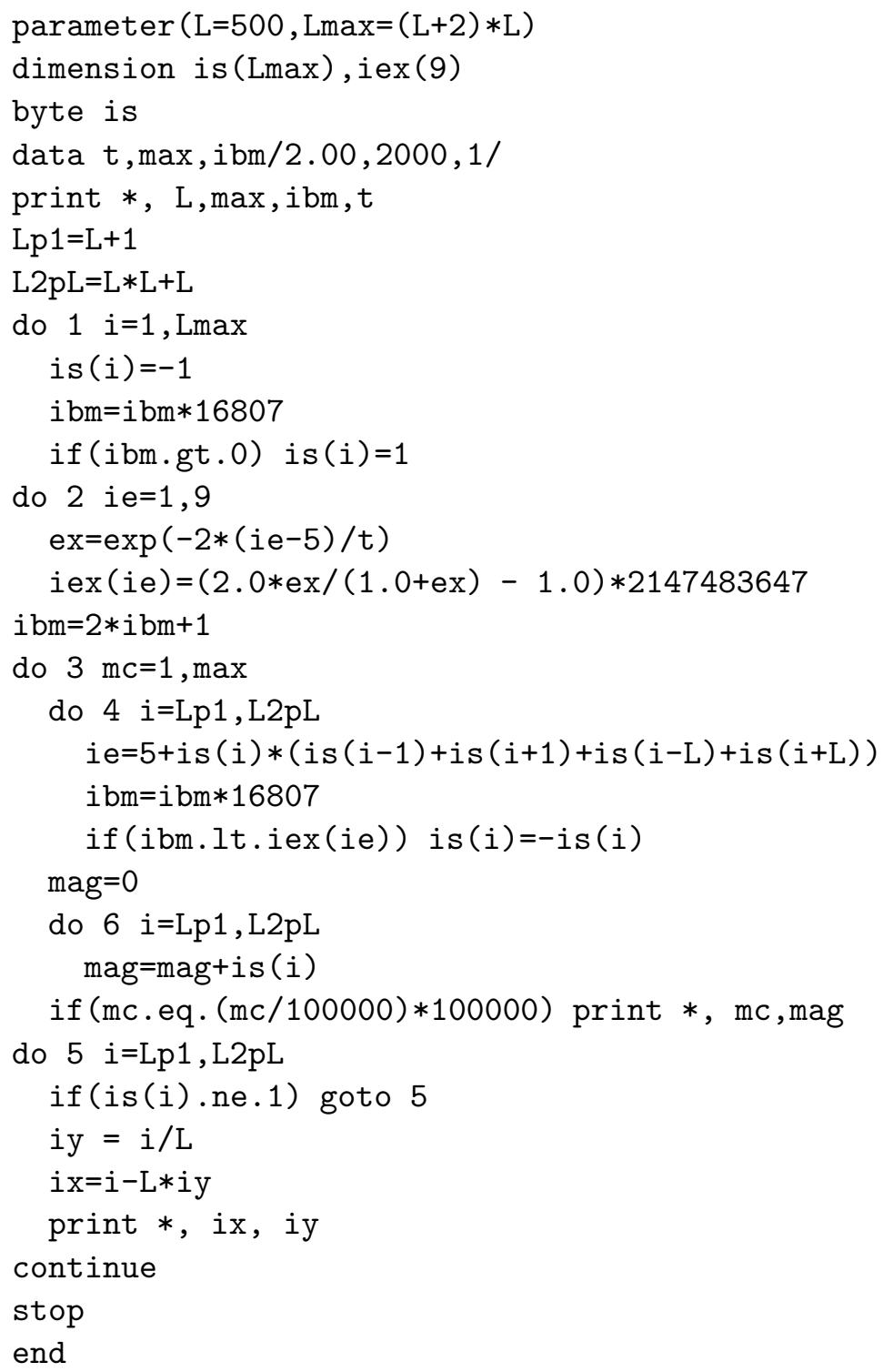

Such models and programs are taught in courses on computational or theoretical physics all over the world; the model was published in 1925. If in the above flipping probability the denominator is omitted one gets the Metropolis kinetics. If instead of flipping one spin we exchange two opposite spins, we get the Kawasaki dynamics. For Glauber or Metropolis, after very long times (measured by the number of sweeps through the lattice) one of the two possibilities dominates at the end, if $T$ is not larger that the critical 
temperature $T_{c}$, with $2 J / k_{B} T_{c}=\ln (1+\sqrt{2}) \simeq 0.44$ known since 1940. For Kawasaki dynamics the fraction of black sites remains constant, and we get two large domains. For higher temperatures above $T_{c}$ only small clusters and no large domains are formed, Fig.3.

Figure 3: As Fig.2 but at $k_{B} T / J=3$ instead of 2. Only small clusters and no large domains are formed. After 200 and 20,000 iterations the pictures look similar to this one made after 2000 iterations.

In this Ising model, two neighbouring spins have due to their interaction $-J S_{i} S_{k}$ a higher probability to belong to the same group than to belong to the two different groups. If the difference between these two probabilities is large enough, $T<T_{c}$, domain sizes can grow to infinity in an infinite lattice, Figs. 1 and 2, while only small clusters are formed in Fig.3 for smaller differences in the probabilities, $T>T_{c}$. That these probabilities, controlled through $-J / k_{B} T$, lead to these different regimes, separated by a sharp phase transition at $T=T_{c}$, is not obvious from the definition of the interaction $J S_{i} S_{k}$, took physicists many years to find, and is typical of complex systems.

The social meaning of temperature $T$ is not what we hear in the weather reports but an overall approximation for all the more or less random events which influence our decisions but are not explicitely included in the model. For residential segregation the model only counts how many neighbours of which group one has. But not all people of one group are alike, housing in different parts of a city costs different amounts of money, some parts are more beautiful then others, and job hunting may force us into a temporary residence of a new city which does not conform to our wishes. In this way, a positive temperature allows for rare moves which increase the energy, i.e. we move to a new residence where the neighbourhood composition along makes us less happy. At zero temperature, the Ising model does not properly order into one or two "infinite" domains.

\subsection{Schelling's version and later improvements}

Schelling [1] avoided probabilistic rules and thus counted neighbours $S_{k}= \pm 1$ at zero temperature. Then it does not matter if all neighbours or only a majority of them belong to the own group. Thus people are defined as happy 
if at least half of the neighbours belong to the own group, and as unhappy otherwise (i.e. if the majority belong to the opposite group). Unhappy people move to the nearest place where they are happy. Since Schelling moved only one person (or family) at a time, and made no exchange of two people simultaneously as in Kawasaki kinetics, he introduces a large fraction of empty residences. Thus at each step, one unhappy person or family moves into the closest vacancy where life would be happy.

This model, and also many variants [1, 3], fail to give large domains; only small clusters are seen. In reality, Harlem in Manhattan (New York), is not a cluster of a few houses but extends over many square kilometers. Thus the original version does not give the desired results. Large domain are formed if people also change residences if this brings no improvement [4] (hardly a realistic assumption) or at a finite temperature [5]. The latter paper also gives some alternatives to the Schelling model which also allow for large domains, and a simple example of s finite cluster where everybody is "happy" and which therefore never grows or dissolves on its own "will".

Much earlier and simpler is the zero-temperature version of Jones [2] who at each iteration removes a random fraction of the people and fills the vacancies with people who are there happy in the Schelling sense. This randomness, just like the finite temperature, leads to large domains as desired. Neither physicists nor social scientists have taken much note of [2]. The history of the Schelling model is an example how the lack of communication between disciplines has hampered progress in research, even very recently [4, 5]. Only computational statistical physicists know everything. (Jones in footnote 4 of [2] also mentions a probabilistic version closer to the Ising model.)

For finite temperatures, 5] follows the above Glauber dynamics, but instead of an energy $E$ uses a variable which is 0 or 1 depending on the happiness of the residents. Moving from one place to the other then depends exponentially on the ratio of this variable to $k_{B} T$, instead of on the ratio $\Delta E / k_{B} T$. Many variants are possible, e.g. in the treatment of neutral cases [4, 6] where the number of neighbours of both groups is exactly the same.

But we are on safer grounds and can use decades of physics research if we use the normal Ising model, or its generalisation to $Q$ different groups, the $Q$-state Potts model. Then [7, 8] implemented a suggestion of Weidlich [9] that people slowly learn to live together with neighbours from the other group. Thus $T$ not only takes into account the various accidents from outside the model, but also measures the tolerance: The higher $T$ is the more are people willing to live in neighbourhoods of the other group. In the limit 
$T=\infty$ the neighbours would not matter at all, for intermediate $T$, Fig.3 showed small clusters but no large domains, and for low $T$ the domains grow to infinite sizes on an infinite lattice. The learning suggested by Weidlich thus means that this parameter $T$ (= temperature or tolerance) no longer is kept constant but slowly increases.

For an Ising model, [7] showed how an initial large domain dissolves if the temperature is slowly increased from below to above $T_{c}$. More realistically, for five (instead of only two) different groups in a modified 5-state Potts model, [8] increased $T$ from low to high values and showed that with a slow increase one has appreciable domain formation during intermediate times, while with a fast increase this segregation is mostly avoided, Fig.4.

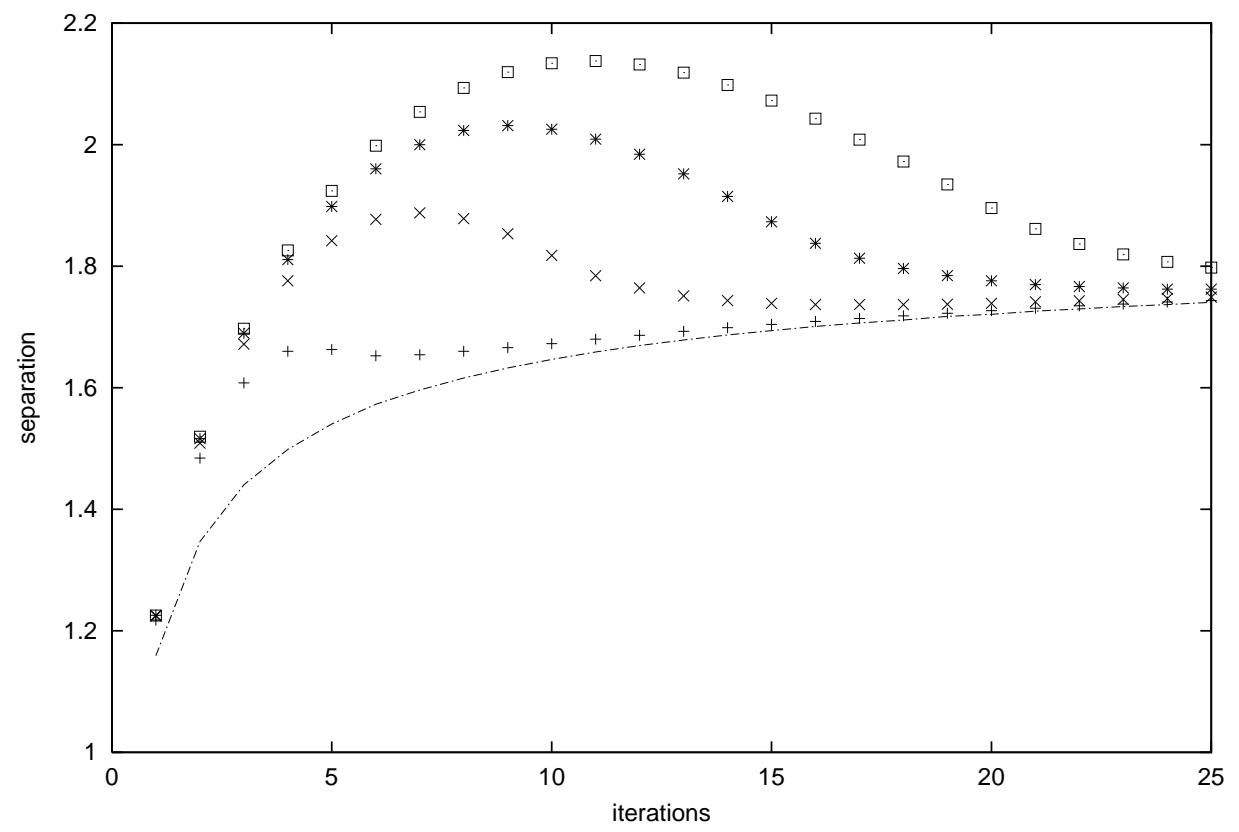

Figure 4: Amount of neighbours of the same type in a Potts model of five groups, normalised to unity for the initial random distribution. The temperature or tolerance increases from low to high values, slowly in the top curves, and fast in the lower curves; the latter mostly avoid the segregation into different group. (The lowest line holds for a constant high temperature.). From [8]. 


\section{Opinion Dynamics}

The following section describes several rules for simulated people to change opinions; each of these rules is applied again and again to these agents until some stationary or static state has been achieved.

\subsection{Ising model}

Also for human opinions, one could use the Ising model of the previous section [10, 11]; see also [9]. People can vote for or against the government or a new constitution, for one of two presidential candidates, or (using generalised models) for one out of $Q>2$ different parties. Their neighbours on a lattice influence them in their vote, and in addition mass media may influence everybody in one direction. The latter effect can be modelled through an external "magnetic" field, eq.(1b) in "Phase transitions ..." by this author in this encyclopedia. No motion of people needs to be taken into account, and the complications of Kawasaki kinetics (exchange of two people with opposing opinions) are not needed. Thus the Glauber program of the previous section still can be used, and we only refer here the old generalisation into the social impact model [12, 13] and to a recent financial application [14].

\section{$3.2 \quad$ Voter model}

Also quite old is the voter model [15]: Each person chooses between two opinions, by taking over the one of a randomly selected neighbour. One may rewrite this rule as stating that each person selects the opinion of the neighbourhood, with a probability proportional to the number $n$ of neighbours having that opinion. Thus in contrast to the Ising model where the probabilities depend exponentially on $n$, now they depend linearly on $n$. A final equilibrium (absorbing fixed point) is reached if everybody shares the same opinion. The deviations from that final state can be measured by the magnetisation (difference between the numbers for the two opinions) or energy (average number of neighbours having the opposite opinion). The time needed to reach the consensus increases with a power of the lattice size, and the exponent depends on the dimensionality. A nice and short review of the voter model, also on various networks, is given by the Majorca group [16]. 


\subsection{Axelrod model}

Axelrod [17] wondered how different opinions or cultures may coexists even if people tend to become more alike in their beliefs. Looking at the above Ising Figs. 1, 2, 3, we see that due to finite time and/or finite temperature such coexistence of two opposing opinions is possible. But Axelrod generalised it not only to $Q>2$ different possible opinions as in the Potts model of the "Schelling" section, but also to $F$ different questions. People may have one set of opinions on which political party they want to vote for, another set about what is the best football team, a third about recent cinema films, etc. This allows for $Q^{F}$ different opinion sets on all $F$ questions ("features"). Of course, one could could generalise this model to the case where the number $Q_{f}$ of possible choices is different for the different features $f$, allowing then for $\Pi_{f=1}^{F} Q_{f}$ instead of simply $Q^{F}$ different sets of opinions.

Another aspect of the model takes into account that people prefer to talk to, or to make political coalitions with, others with whom they share many opinions. Thus the probability of one person to take over the different opinion of a neighbour is proportional to the number of features on which their opinions already agree. In the next subsection we will use a similar concept under the name of bounded confidence.

Whether a total consensus ("globalisation") is reached or multiculturality persists depends on parameters: Small $Q$ lead to consensus. Again, the Majorca group [16] reviewed the many follow-up papers on this Axelrod model.

\subsection{Sznajd, Krause-Hegselmann and Deffuant models}

Much of the opinion dynamics research since 2000 centred on three different models $\mathrm{S}, \mathrm{KH}$ and $\mathrm{D}$, originally invented independently around that year: Sznajd [18] (S), Krause-Hegselmann [19] (KH) and Deffuant et al [20] (D). They were also called missionaries, opportunists and negotiators by some computational physicists [39].

The $\mathrm{S}$ model is closest to the earlier models since it allows for $Q$ discrete opinions, while $\mathrm{KH}$ and $\mathrm{D}$ use real opinions, e.g. between zero and one. S happens on a lattice or network while for $\mathrm{KH}$ and D everybody may interact with everybody. In the most widespread $\mathrm{S}$ version a pair of neighbouring sites on a square lattice convinces its six neighbours of its opinion, if and only if the two opinions of the pair agree [21]; governments and parties usually 
lose support if their internal opinion differences make it to the headlines. For $\mathrm{KH}$, the new opinion of a person is the arithmetic average over the opinion of the whole population. For D, each person selects randomly another person and then both move in their opinion towards each other by an amount proportional to their opinion difference.

In all three cases, "bounded confidence" applies: The KH agents average only over those people who differ from their own opinion by less than $\epsilon$, and the $\mathrm{D}$ agents only select negotiation partners differing by less than $\epsilon$ from their own opinions. In both models $0<\epsilon<1$ is a fixed parameter. For $S$ agents with $Q=2$ such a rule makes no sense, but for $Q>2$ one can modify the convincing rule such that only neighbours differing by at most \pm 1 from the pair opinion adopt the pair opinion. Thus $1 / Q$ for $\mathrm{S}$ plays the role of $\epsilon$ for D and KH. A rule similar to this bounded confidence was mentioned above for the Axelrod model [17].

Inspite of the differences in their definitions, the results are quite similar for $\mathrm{S}, \mathrm{KH}$ and $\mathrm{D}$. For large $\epsilon$ or $Q \leq 3$ a complete consensus is usually reached; for small $\epsilon$ or $Q \geq 4$ different opinions may coexist forever. In addition to computer simulations, also analytical calculations were made [22, 23] which agree with many aspects of the simulations. More results, also for opinions on more than one feature and agents sitting on scale-free networks, are summarised by us in 39].

One particular application is shown in Fig.5: Various Brazilian election results for candidates in city councils showed great similarity if the number of candidates getting a given number $v$ of votes is plotted against this $v$. Putting the $S$ model with $Q=1000$ candidates onto a scale-free network instead of a square lattice, excellent agreement of simulation and reality was found after the numbers were scaled by suitable factors. Also Indian elections were simulated this way [25], while some exceptions may also exist (S. Fortunato, unpublished). It would be nice to apply other opinion dynamics models to the same election problem. As usual in statistical physics, these studies can predict and simulate the shape of the distributions but not the winner in a specific election, just as we can predict the pressure of the air molecules around us but not where which molecule will be one minute from now.

\subsection{Galam conservatism}

Galam has published since many years theoretical models which may explain why reforms are very difficult and why a minority can stay in power. Usu- 


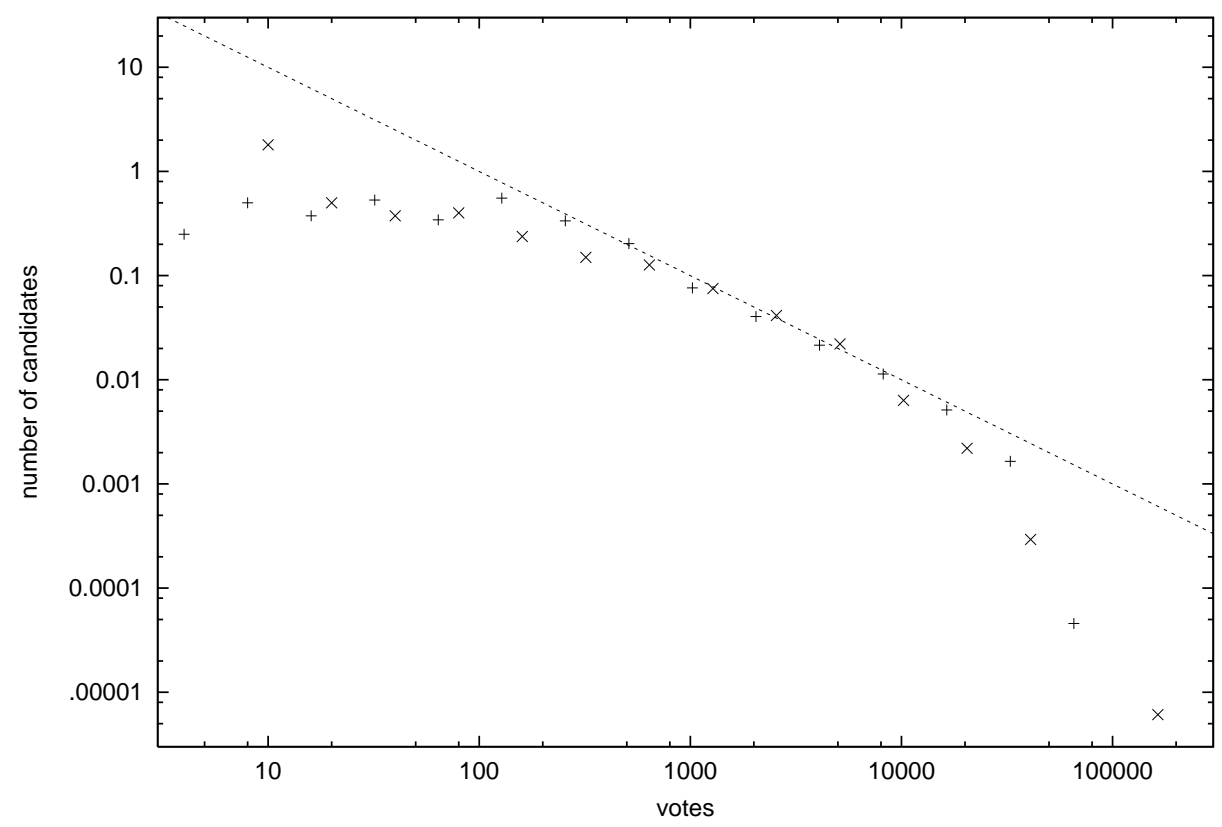

Figure 5: Brazilian elections (x) and simulations of Sznajd model on Barabási - Albert networks (+); from [24].

ally these models are solvable analytically and assume that the population is divided into small groups of people which to the outside are represented by one person who follows the majority wish of the group. Several of these representatives form a supergroup, and this supergroup again decides according to the majority of the representatives in it. In this way an "infinite" hierarchy of people, groups, supergroups etc can be built. In the case of equally many voting for one choice as for the opposite choice, within one unit, that unit votes for the status quo. Starting with everybody having opinion -1 , a very large majority of people must switch to opinion +1 before the top of the hierarchy finally also changes opinion [26]. We refer to our book [39] for a summary of more recent Galam papers. 


\section{Languages, Hierarchies and Football}

\subsection{Language competition}

Darwinian survival of the fittest is established biology, but similar concepts can be applied to human languages, bridging the gap to opinion dynamics. There are now thousands of different languages, and their "size" is the number of native speakers of that language. The size distribution extends from 1 (on the verge of extinction) to $10^{9}$ (Mandarin Chinese). The grammar of a language 27] can be characterised by $F$ features each of which can have $Q$ different values, just as in Axelrod's model explained above. Features can change spontaneously or be taken over from a (neighbouring) language; speakers of a small language give it up and learn a widespread language (as done with physics research publications since 1945); people migrate to other places and bring their language with them. All these processes can lead to the extinction of existing languages and the creation of new ones (by the branching of one language into several daughter languages.) The present language size distribution is roughly log-normal, with an enhancement at small sizes [28]. Similar languages form families, and the size distribution of families is a power law at intermediate sizes [29] (where the size is now the number of different languages) belonging to that family).

Various computer simulations of this language competition have been made, mostly since 2003 and reviewed recently [30]; see also [31, 32]. We only mention Fig.6 from a modified Viviane model which agrees well with the real language size distribution. For language families, the empirical statistics is worse [29] and the models work less well [30].

\subsection{Self-organisation of social hierarchies}

The elites of all countries and all times always had excellent reasons why they should be on top and others on the bottom. This holds even when the United Nations criticise the school system as violating human rights. In contrast, the Bonabeau model [34] explains social hierarchies as purely accidental, without any merit. People are put on a lattice, occupying a fraction $p$ of all lattice sites and having an initial score of zero. Then they move randomly to neighbouring sites, and whenever one person wants to move into the site occupied by another person, a fight erupts. The winner takes the contested site, the loser moves into (or stays at) the other site. Also, the winner adds 


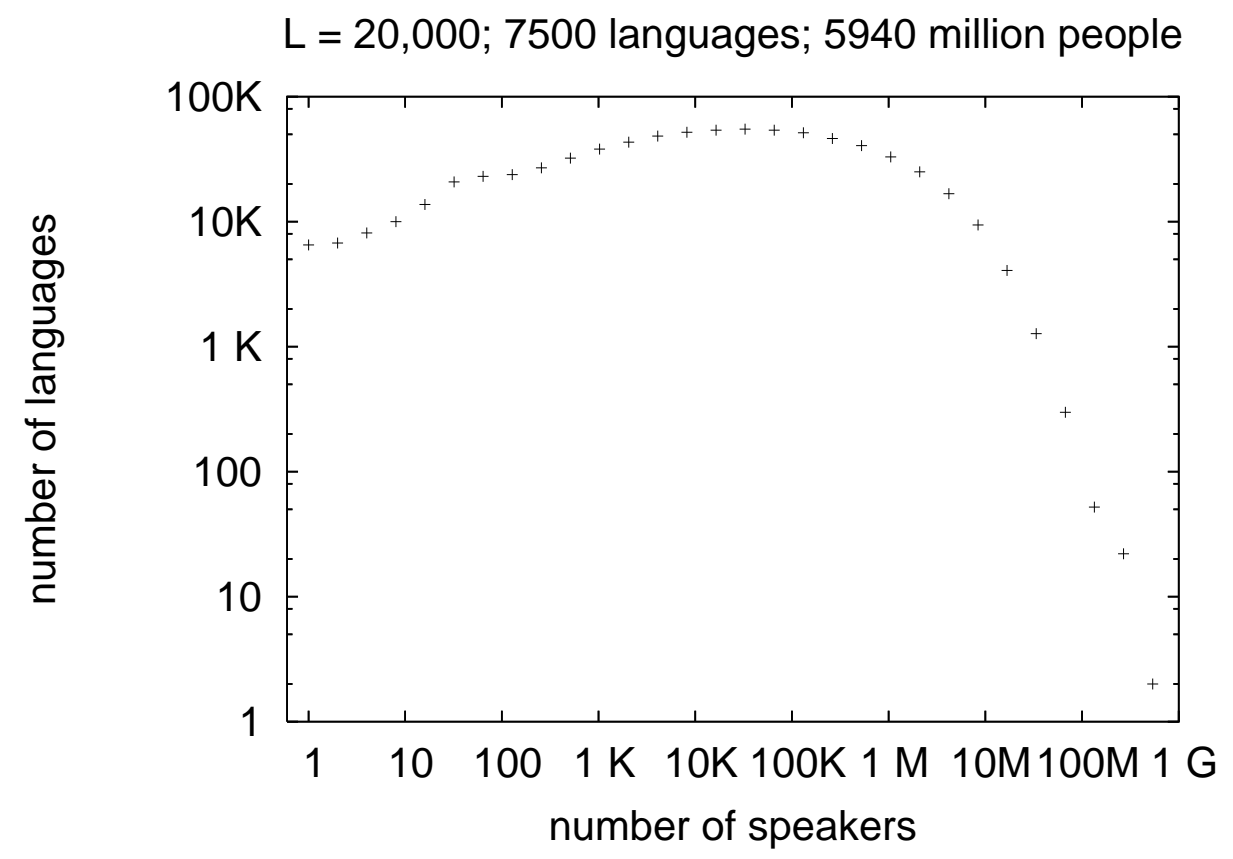

Figure 6: Simulated language size distribution on a $20,000 \times 20,000$ square lattice using a modified Viviane model [33].

one point and the loser subtracts one point in its score, and in the future the agents with a positive score have a higher probability to win, those with a negative score have a lower probability to win. Slowly the history is forgotten, by reducing the score at each time step by, say, ten percent.

With some suitable feedback between the distribution of scores and the probability to win, a phase transition was simulated such that for $p$ above some critical concentration, the standard deviation in the scores becomes positive for long times and large populations. For $p<p_{c}$ it fluctuates near zero, that means everybody remains near a 50 percent chance to win. So, just by accident at a high population density some people rise to the top, and others fall to the bottom. However, the people on top (bottom) are not always the same; only the differences between top and bottom, not the people, remain the same. [35, 36, 37] are some of the more recent references in this field. 


\subsection{Football}

Football (= soccer) is the world's most popular spectator sport, though in the author's city it is more a frustration. Randomness surely plays a role and makes it attractive. Can we explain all results just by chance, in the spirit of Bonabeau hierarchies? Assuming a constant probability to make a goal within one minute, the distribution of goals and victories is more narrow than in reality. If instead we assume that this probability varies from team to team, still no good agreement is found. Good agreement with reality is obtained only if correlations are taken into account [38, in the sense that a goal makes the scoring team happy, shocks the opposing team, and thus with an enhanced probability leads to another goal for the scoring team. Thus if we lose it is not just bad luck; it is also the referee's fault.

\section{$5 \quad$ Future Directions}

The Schelling model of section 2 is not the only case of missed opportunities because of a lack of cooperation between social sciences on the one side and physics, mathematics or computer science on the other side. The two books cited at the end [39] were written without the authors of one book knowing of the preparation of the other book. One group of authors works in physics departments; none of the other group lists physics as institutional address. Nevertheless the two books show strong overlap in fields and methods covered, but little overlap in the literature cited. More interdisciplinary cooperation would help.

\section{References}

\section{[1] Primary literature:}

Schelling TC (1971) J. Math. Sociol. 1: 143

[2] Jones FL (1985) Aust. NZ J. Sociol. 21: 431

[3] Fossett M (2006) J. Math. Sociol. 30: 185

[4] Vinkovic D, Kirman A (2006) Proc. Natl. Acad. Sci. USA 103: 19261 
[5] Stauffer D, Solomon S (2007) physics/0701051 at arXiv.org, submitted to Eur. Phys. J. B

[6] Müller, K. (2007) bachelor thesis, Cologne University

[7] Meyer-Ortmanns H (2003) Int. J. Mod. Phys. C 14: 311

[8] Schulze C (2005) Int. J. Mod. Phys. C 16: 351

[9] Weidlich W (2000) Sociodynamics; A Systematic Approach to Mathematical Modelling in the Social Sciences Harwood Academic Publishers; 2006 reprint: Dover, Mineola (New York)

[10] Callen E, Shapero D, Physics Today (July 1974) 27: 23

[11] Galam S, Gefen Y, Shapir Y (1982) J. Mathematical Sociology 9: 1

[12] Latané B (1981) Am. Psychologist 36: 343

[13] Hołyst JA, Kacperski K, Schweitzer F (2001), p.275 in Annual Reviews of Computational Physics IX, World Scientific, Singapore

[14] Zhou WX, Sornette D (2007) Eur. Phys. J. B 55: 175

[15] Liggett TM (1985) Interacting Particle Systems, Springer, New York

[16] San Miguel M, Eguíluz VM, Toral M (Nov/Dec 2005) Computing Sci. Engin 7: 67

[17] Axelrod R (1997) J. Conflict Resol. 41: 203

[18] Sznajd-Weron K, Sznajd J (2000) Int. J. Mod. Phys. C 11: 1157

[19] Hegselmann R, Krause U, 2002, Journal of Artificial Societies and Social Simulation 5 (3): 2 (jasss.soc.surrey.ac.uk)

[20] Deffuant G, Amblard F, Weisbuch G, Faure T (2002) Journal of Artificial Societies and Social Simulation 5 (4): 1 (jasss.soc.surrey.ac.uk).

[21] Milgram S, Bickman L, Berkowitz L (1969) J. Pers. Soc. Psych. 13: 79

[22] Ben-Naim, E, Krapivsky P, Redner S (2003) Physica D 183: 190

[23] Slanina F, Laviçka H (2003) Eur. Phys. J. B 35: 279 
[24] Bernardes, AT, Stauffer D, Kertész J (2002) Eur. Phys. J. B 25: 123

[25] González MC, Sousa AO, Herrmann HJ (2004) Int. J. Mod. Phys. C 15: 45

[26] Galam S (1990) J. Stat. Phys. 61: 943

[27] Haspelmath M, Dryer M, Gil D, and Comrie C (eds.) (2005) The World Atlas of Language Structures. Oxford: Oxford University Press

[28] Grimes BF (2000) Ethnologue: Languages of the World (14th edn.) Dallas, TX: Summer Institute of Linguistics; and www.ethnologue.org

[29] Wichmann S (2005) J. Linguistics 41: 117

[30] Schulze S, Stauffer D, Wichmann S (2007) 0704.0071 at arXiv.org; submitted to Commm. Comput. Phys.

[31] Cangelosi A, Parisi D (eds.) (2002) Simulating the Evolution of Language. Springer, New York

[32] Culicover P, Nowak A (2003) Dynamical Grammar, Oxford University Press, Oxford

[33] de Oliveira PMC, Stauffer D, Lima FWS, Sousa AO, Schulze C, Moss de Oliveira S (2007), Physica A 376: 609

[34] Bonabeau E, Theraulaz G, Deneubourg J-L (1995) Physica A217: 373

[35] Ben-Naim E, Vazquez F, Redner S (2006) Eur. Phys. J. B 49: 531

[36] Naumis GG, del Castillo-Mussot M, Perez LA, Vazquez GJ (2006) Physica A 369: 789

[37] Weisbuch G, Stauffer D (2007) Physica A, in press

[38] Bittner E, Nussbaumer A, Janke W, Weigel M (2007) Europhys. Lett., in press.

[39] Books and Reviews: 
Stauffer D, Moss de Oliveira S, de Oliveira PMC, Sá Martins JS (2006) Biology, Sociology, Geology by Computational Physicists, Elsevier, Amsterdam

Billari FC, Fent T, Prskawetz A, Scheffran J (2006) Agent-based computational modelling, Physica-Verlag, Heidelberg 